\title{
Image Processing for Phase Imperfections Reconstructed in Electron Holography
}

\author{
Wei $\mathrm{Li}^{1}$, Takayoshi Tanji ${ }^{2}$ \\ 1. Graduate School of Engineering, Nagoya University, Japan \\ 2. EcoTopia Science Institute, Nagoya University, Japan
}

Electron holography is an ideal technique and a powerful tool for observing nanoscale electromagnetic fields [1]. Today, most holograms are reconstructed by the Fourier transform method [2,3]. In some cases, however, a phenomenon in which the contrast reverses abruptly from white to black may occur in phase images directly reconstructed by digital Fourier transform. Two types of abrupt reversal occur in the phase contrast images. One is the well-known phase jump of $2 \pi$ [4] during the digital reconstruction process, caused by the reconstructed phase falling within the range of $[-\pi, \pi][5]$. In this case, the phase can be unwrapped [6] and smoothly connected for such a jump using the appropriate software. The other type of reversal in the reconstructed phase image is caused by the disconnection of interference fringes due to the weak electron-wave amplitude in specific areas of the specimen. In the article, we propose a method for removing the influence of such singularities on the reconstructed phase by using image processing in electron holography.

The interference pattern of an electron hologram of a specimen was captured by a slow-scan CCD camera (Gatan; Model 794) attached to the bottom of a Hitachi field-emission electron microscope (HF-2000) operated at $200 \mathrm{kV}$. An electron biprism was positioned in the image plane to make an electron interferogram. First, the image containing only the interference fringes was reproduced by using a low-pass filter to reduce the influence of Fresnel diffraction and noise [7]. Then, binarization was performed to obtain a binary image of the interference fringes [8]. Skeletonization and burr removal of the binary image allowed us to obtain the interference fringes with a width of only one pixel. The singularities disconnected or incorrectly connected are searched out using the skeletonized fringes based on mathematical morphology. Finally, we correct the failed interference fringes, which are mainly disconnected or incorrectly connected, by cut-and-reconnect processing using MATLAB (MathWorks). The entire procedure is illustrated in Fig. 1.

An electron hologram of a $\mathrm{CeO}_{2}$ particle captured by a CCD camera is shown in Fig. 2(a). The reconstructed phase image obtained directly from the electron hologram is shown in Fig. 2(b), including some phase imperfections indicated by arrowheads. A binary image of the interference fringes can be used to reconstruct the phase images; nevertheless, imperfections in the interference fringes, namely disconnected or incorrectly connected fringes, occur in the binary image, as shown in Fig. 2(c). The distorted interference fringes in Fig. 2(c) are corrected by cut-and-reconnect processing, and then the corrected interference fringes are smoothed out by using a median filter, as 
shown in Fig. 2(d). The reconstructed phase image in Fig. 2(e) obtained from the corrected interference fringes shows that the phase error due to the weak electron-wave amplitude has been drastically reduced. The unwrapping process is taken to remove the phase jump due to the digital reconstruction process to obtain the final image without any phase jump, as shown in Fig. 2(f). The phase image reconstructed from the corrected interference fringes showed that the phase error due to the weak electron-wave amplitude in the specimen was drastically reduced by using the proposed method.

\section{References}

[1] T. Hirayama, Q. Ru, T. Tanji and A. Tonomura. Jpn. J. Appl. Phys. 63: 418 (1993)

[2] M. Lehmann and H. Lichte. Electron Holography. Elsevier, Amsterdam (1995)

[3] K. Ishizuka. Ultramicroscopy 51: 1 (1999)

[4] D. J. Smith, W. J. de Ruijiter, M. R. McCartney and J. K. Weiss. In: E. Völkl, L. F. Allard and D. C. Joy eds. Introduction to Electron Holography. Kluwer, New York (1998)

[5] A. Tonomura. Electron Holography. Springer-Verlag, Berlin (1999)

[6] D. C. Ghiglia and M. D. Pritt. Two-Dimensional Phase Unwrapping: Theory Algorithms and Software. Wiley, New York (1995)

[7] T. Tanji, H. Hashimoto, H. Endoh and H. Tomioka. J. Electron Microscopy 31:1 (1982)

[8] R. C. Gonzalez and R. E. Woods. Digital Image Processing (2002)

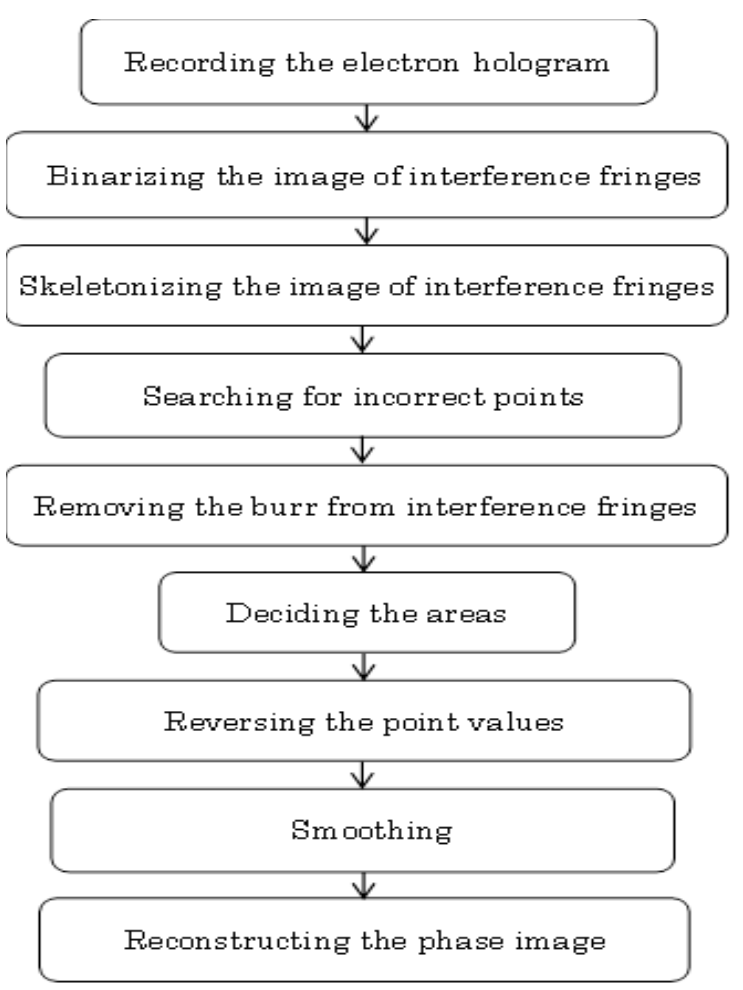

Fig. 1. Flow chart of the process. The process can be described with nine steps.

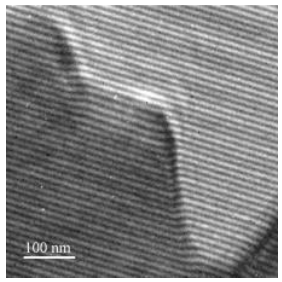

(a)

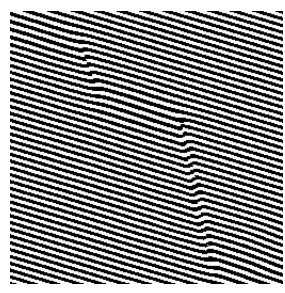

(d)

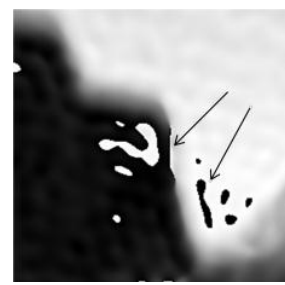

(b)

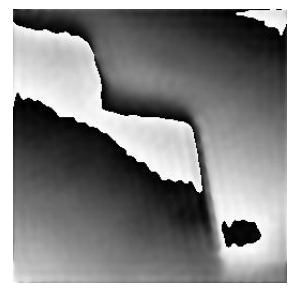

(e)

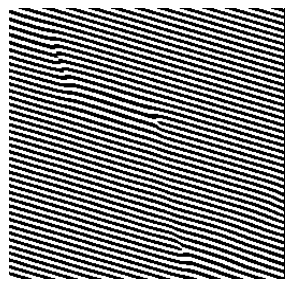

(c)

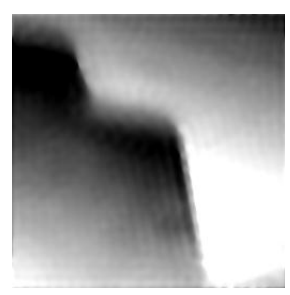

(f)
Fig. 2. Images of the results. (a) Corrected fringes; (b) Corrected fringes after smoothing; (c) Reconstructed phase image; (d) Corrected fringes after smoothing; (e) Reconstructed phase image; (f) Final phase image. 